Gut, 1962, 3, 38

\title{
Medical management of chronic gastric ulcer
}

\author{
DAVID FERRIMAN
}

From North Middlesex Hospital, Edmonton, London

SYNOPSIS The results of medical treatment in 113 patients with chronic ulceration of the angulus and vertical part of the stomach are reported. The ulcers failed to heal in $38 \%$ and healed but recurred in $22 \%$. The ulcers healed and, except for minor recurrences in a few, remained healed for five years in $40 \%$. The prognosis for patients with ulcers with long histories and large ulcers was poor, and this is true of other series reported in the literature. If such cases had been excluded from the present series the success rate would have risen to $64 \%$. Patients with ulcers in these two categories should be referred for surgery. It is suggested that a trial of medical treatment is justifiable in most other cases, surgery being reserved for its failures.

The patients concerned in this study all came under the care of one general physician at the North Middlesex Hospital between the years 1947 and 1952 inclusive. They were first seen either at a general medical out-patient clinic, or were admitted with acute exacerbated dyspepsia, or following gastrointestinal haemorrhage, to one male and one female general medical ward. Special attention was paid to a number of factors which might bear on the prognosis for medical treatment.

\section{MATERIAL AND METHODS}

CASE MATERIAL All patients with ulcers clearly visible on radiographs were included in the study, with the following exceptions:-

1 Patients admitted with haemorrhage for whom an emergency operation was performed shortly after admission; 2 , patients with pyloric stenosis, hour glass stomach, concomitant antral or duodenal ulceration, and a previous partial gastrectomy or gastro-enterostomy; 3 , patients who had recently received an adequate course of medical treatment (4) or declined to accept such a course (18); and 4, patients over the age of 67.

This resulted in 121 patients coming under study. Eight left the district, or died from causes unconnected with ulcers before the follow-up was complete, and have been excluded from an analysis of results. Follow-up was successfully achieved in all of the remaining 113 .

DETAILS OF TREATMENT Patients were treated for three months in hospital, or for six weeks in hospital followed by six weeks at home. They were allowed up to the lavatory only in the first month, for two hours a day only in the second month, and for half a day daily in the third. A milk-alkali drip was given for 22 hours daily in the first fortnight. Thereafter they were placed on a bland gastric diet by day, with a continuation of the drip by night for the first two weeks. Midway between each feed the patient took one drachm of Aludrox. Attention was paid to psychological factors. On discharge the patients continued with their diet and alkalis until the ulcer was thought to be healed, when some relaxation of diet was permitted, and alkalis were discontinued except on any return of symptoms.

FOLLOW-UP ARRANGEMENTS Barium meals were performed at six weeks, three months, and six months from the start of treatment, and if necessary at six-monthly intervals thereafter. Three months after the first barium meal to show healing a gastroscopy was performed, and this was repeated at three- to six-monthly intervals as seemed appropriate until the ulcer was completely healed. Patients were then followed at six-monthly or yearly intervals and were also instructed to report on the recurrence of significant symptoms. Barium meals were requested when this seemed desirable. Follow-up ceased in those referred for surgery. It was continued successfully for five years in the remainder (except for those dying or leaving the district).

DECISION TO OPERATE Surgery was advised under the following circumstances:-

1 Occurrence of haemorrhage or perforation at any time after the onset of medical treatment; 2 significant persistence of pain beyond a fortnight; 3 lack of visible radiological improvement in large ulcers at the end of six weeks, and in any at the end of three months; 4 persistence of significant ulceration shown radiologically at the end of six months; or 5 gastroscopically at the end of one year, except where distinct improvement on previous gastroscopy had taken place; 6 recurrence after healing of the original ulcer.

\section{RESULTS}

The ulcers failed to heal in $43(38 \%)$. The ulcers 
healed but frankly recurred in $25(22 \%)$. The ulcers healed and except for minor recurrences in a few remained healed in $45(40 \%)$.

FAILURE TO HEAL It seemed of interest to analyse the reasons behind the decision to resort to surgery, and the times at which these decisions occurred. Four main periods were recognized. These periods, the reasons for decision, and the numbers involved are shown in Table I.

\section{TABLE I}

REASONS AND TIMES FOR DECISION TO OPERATE

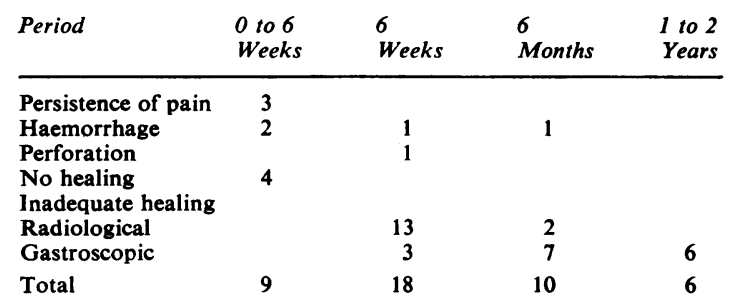

The patient with perforation was found to have an enormous irremovable ulcer; he died later from haematemesis. Another patient with a large ulcer was offered operation but refused, experienced a haematemesis at six months, and eventually accepted operation at the end of 18 months. A third patient refused operation and died later from haematemesis.

Three other patients refused operation and one was considered unsuitable. Partial gastrectomy was eventually performed on 37 patients; there were two operative deaths.

RECURRENCES Table II shows the year from start of treatment in which recurrence took place.

\section{TABLE II}

TIME OF RELAPSE

\begin{tabular}{cccccc} 
Time in Years & First & Second & Third & Fourth & Fifth \\
\hline Patients & 0 & 8 & 9 & 4 & 4
\end{tabular}

Three recurrences were with haematemesis, from which one patient died; this death must be regarded as due to medical management, the only one in the series of 113 patients. Operation could not be performed in seven, and was refused by five. Partial gastrectomy was performed in 13 cases; there were no deaths.

SUCCESSES All but two of the ulcers in the successfully treated group are known to have healed by the end of one year, and one of these two had healed by
13 months. Table III shows these 45 patients graded according to the degree of success, the grades corresponding approximately to those in Visick's useful classification (1948). The first group consists of those with no or trivial dyspepsia. Rather more than trivial dysepsia occurred in the second group but this did not interfere with work or with the enjoyment of life, and barium meals showed no abnormality. The third group consists of five patients with short-lived radiological recurrences, and two who experienced small haematemeses with no demonstrable ulcer in one, and a small ulcer in the other. It was not considered necessary to abandon treatment in these patients.

\section{TABLE III}

\begin{tabular}{|c|c|c|c|}
\hline & & SUCCESS & \\
\hline Grade & 1 & 2 & 3 \\
\hline Number & 26 & 12 & 7 \\
\hline
\end{tabular}

FACTORS BEARING ON PROGNOSIS A number of factors have been studied for their bearing on prognosis.

Length of ulcer history Scrutiny of the results showed a worsening prognosis from the third year onwards. A grouped analysis of the figures is set out in Table IV. Failures include recurrences.

\section{TABLE IV}

FAILURES IN RELATION TO LENGTH OF HISTORY

\begin{tabular}{llll} 
Duration in Years & 0 to 2 & $2-$ & $10-$ \\
\hline Total & 49 & 32 & 32 \\
Failures & 22 & 22 & 24 \\
$\%$ Failures & 45 & 69 & 75
\end{tabular}

Using the $\chi^{2}$ test for a trend (Armitage, 1955), the worsening prognosis with length of history is very significant $(1 \%)$. The recurrence rate in patients with ulcer histories of over two years is twice as great as those with shorter histories, suggesting that a tendency to recurrence underlies these findings.

Ulcer size Ulcers were graded on radiological appearance as large, medium, and small, approximating to diameters of $\frac{1}{4}$ in., $\frac{1}{2}$ in., and 1 in. respectively. The failure rates (including recurrences) are set out in Table V. Only 110 cases were available for analysis, since ulcer sizes were not recorded in three.

TABLE V

FAILURE IN RELATION TO ULCER SIZE

\begin{tabular}{lccc} 
Size & Small & Medium & Large \\
\hline Total & 45 & 48 & 17 \\
Failures & 23 & 28 & 14 \\
$\%$ Failures & 51 & 58 & 82
\end{tabular}


Clearly prognosis for large ulcers is poor. The difference between small and medium-sized ulcers is less obvious. Using the $\chi^{2}$ test for a trend (Armitage, 1955), the worsening prognosis with size of ulcer is just significant $(5 \%)$. The poor prognosis in large ulcers is mainly accounted for by an initial failure of healing ( $80 \%$ as compared with $55 \%$ for the rest).

Correlation between ulcer size and length of histories Analysis failed to show any such correlation. Indeed, a particularly high incidence of short histories was found in the patients with large ulcers.

Sex Men fared worse than women, success being achieved in about one-third ( 27 out of 78 ) compared with about half the women (18 out of 35 ).This could not be accounted for by a lesser number of large ulcers or long histories in the women.

Age of patient After allowance for the length of history, age still seemed something of an adverse factor, with 45 as an approximate dividing line. The numbers involved did not permit of statistical analysis.

Family history The presence or absence of a family history rather surprisingly failed to show any difference in prognosis.

Long histories and large ulcers were bad prognostic features. Masculine sex certainly, and an age over 45 probably, were adverse factors. Family history showed no significance in this series.

MisCELLANEOUS OBSERVATIONS Several miscellaneous aspects deserve consideration.

Persistence of ulceration It is well known that ulcers can persist unhealed for long periods of time. The present series illustrates this clearly. In the 113 patients, ulcers remained unhealed for over six months in 23, from six months to a year in 15 , from one to one and a half years in four, and from one and a half to two years in four.

Emphysema and gastric ulceration It is of interest that nine of the 113 patients, including five of the 25 with recurrences, suffered from emphysema. The association has been noted by several authors (Avery Jones, 1957; Flint and Warrack, 1958).

Value of gastroscopy It was thought that the recurrence rate following gastroscopic demonstration of healing would have been less than that following radiological healing, since the latter might include relapses of unhealed ulcers. In fact the recurrence rates turned out to be much the same.

\section{DISCUSSION}

It is proposed to compare the results of treatment in this and other series from the literature, and to discuss their bearing on the management of chronic gastric ulcer.

PROGNOSIS FOR MEDICAL TREATMENT Findings may be considered under several headings.

Overall success rate An attempt has been made to determine the proportion of cases in similar series from the literature corresponding with the successfully treated group as a whole in the present series, and also with that proportion of it falling into grade 1 . The findings are set out in Table VI.

\section{TABLE VI}

PERCENTAGE OF SUCCESSES

\begin{tabular}{lcc} 
Authors & Grade 1 & All Grades \\
\hline Qvigstad and Römcke (1946) & 22 & 40 \\
Malmros and Hiertonn (1949) & 22 & $48 \cdot 5$ \\
Martin and Lewis (1949) & 24 & 42 \\
Swynnerton and Tanner (1953) & & 87 \\
Finer and Fry (1955) & 23 & 40 \\
Ferriman (present series) & &
\end{tabular}

A very striking correspondence of figures will be observed for those falling into grade 1 . This suggests that about one-fifth of all chronic gastric ulcers may be non-recurring in nature.

A fair correspondence is observed for the success group as a whole, except for the series reported by Finer and Fry (1955). This report concerned cases encountered in general practice, and suggests that the prognosis for such cases is better than for cases met with in hospital practice. It may be concluded that reasonably satisfactory results may be expected in rather more than one-third of cases in hospital practice.

Length of history The poor prognosis in patients with a long history of dyspepsia seems to be well established. It is seen clearly in the present series and has been observed also by Qvigstad and Römcke (1946), Malmros and Hiertonn (1949), Martin and Lewis (1949), Barsby (1951), and Swynnerton and Tanner (1953). It is perhaps only to be expected, since a long history may reflect a tendency to recurrence.

Size of ulcer It has been customary in the past to operate for large ulcers on the assumption that many are malignant, a danger which seems to have been overestimated (Pygott, 1958). A relative paucity of data exists therefore for the prognosis of medical treatment in this group. However, patients with such ulcers have fared badly in the present series and in those reported by Steigmann (1943), Branwood (1950), Jennings and Richardson (1954), and Strange (1959). The proportion of failures (four out of five) in Strange's series of 37 patients is 
particularly discouraging. The incidence of haemorrhage and perforation seems particularly serious; Branwood (1950) reported five deaths in 36 patients who showed radiological healing, a mortality rate of nearly $15 \%$.

Haemorrhage and perforation The risks from haemorrhage and perforation following a decision to employ medical treatment in the present series, and in similar series from the literature, are reviewed in Table VII.

\section{TABLE VII}

INCIDENCE AND MORTALITY FROM HAEMORRHAGE AND PERFORATION AFTER MEDICAL TREATMENT

\begin{tabular}{|c|c|c|c|c|}
\hline & \multicolumn{2}{|c|}{ Haemorrhage } & \multicolumn{2}{|c|}{ Perforation } \\
\hline & $\begin{array}{l}\text { Incidence } \\
(\%)\end{array}$ & $\begin{array}{l}\text { Mortality } \\
(\%)\end{array}$ & $\begin{array}{l}\text { Incidence } \\
(\%)\end{array}$ & $\begin{array}{l}\text { Mortality } \\
(\%)\end{array}$ \\
\hline \multirow{4}{*}{$\begin{array}{l}\text { Natvig, Römcke, and } \\
\text { Svaar-Seljesaeter (1943) } \\
\text { Malmros and Hiertonn } \\
\text { (1949) } \\
\text { Martin and Lewis } \\
\text { (1949) } \\
\text { Finer and Fry (1955) } \\
\text { Ferriman (present } \\
\text { series) }\end{array}$} & 3 & 0 & 1 & 0 \\
\hline & & 2 & 3 & 1 \\
\hline & 19 & 0 & 0 & 0 \\
\hline & 11 & 1 & 1 & 0 \\
\hline
\end{tabular}

Although there were three deaths in the present series, only one need be regarded as a failure of medical management; a second patient had refused operation, and the other had been found to have an irremovable ulcer at a previous operation. However, the risk from haemorrhage and perforation is obviously very real and may be in the order of $2 \%$. This risk must be set against the danger from operation. The latter however, is less than $1 \%$ in specialized clinics (Avery Jones, 1959), though it may be higher elsewhere.

Malignant ulcers The danger of mistaking malignancy for simple ulcer appears to have been considerably overestimated in the past (Doll, Jones, Pygott, and Stubbe 1957). No such case was encountered in the present series. Provided a competent radiologist is available, it would seem that the possibility should not influence decision unduly.

MANAGEMENT OF CHRONIC GASTRIC ULCER The limited success rate from medical treatment and the danger from subsequent haemorrhage and perforation suggest that medical treatment has a limited part to play. However, the success rate is not negligible and could moreover be much improved if cases with a demonstrably poor prognosis were excluded. It is worth noting that if all patients in the present series with a history of over four years' duration or large ulcers had been referred initially for surgery, the proportion of successes in the then medically treated group would have risen from $40 \%$ to $64 \%$. Moreover, surgical treatment is not without a mortality rate, though small in expert hands, and has drawbacks of its own such as the development of anaemia and the dumping syndrome.

It would seem reasonable to suggest that cases with an unfavourable prognosis, such as a long history of dysepsia and large ulcers, should be referred initially for surgery. Factors such as the frequency and duration of bouts of dyspepsia must be studied in relation to the length of history, but it would seem desirable to consider surgery with histories of three to five years or upwards. It would seem reasonable to make a trial of medical treatment in cases with a more favourable prognosis and to reserve surgery for its failures. If medical treatment is employed, it should be thorough. It is not proposed to specify this further, except to note that admission to hospital is beneficial (Doll and Pygott, 1952).

I am indebted to many members of the nursing staff, health visitors, house physicians, and medical registrars for assistance in this study. In particular I am indebted to Miss M. Jones, one of the lady almoners, who was largely responsible for the successful tracing of patients; Dr. T. E. Broadbent, Dr. M. M. Craig, and Dr. E. E. Houldsworth, the radiologist who carried out the many barium meal examinations; Mr. M. P. Curwen for statistical advice; and Dr. Avery Jones for helpful suggestions and for reading the manuscript.

\section{REFERENCES}

Armitage, P. (1955). Tests for linear trends in proportions and frequencies. Biometrics, 11, 375 .

Barsby, B. (1951). Prognosis of healed gastric ulcers. Lancet, 2, 59. Branwood, A. W. (1950). The large gastric ulcer. Edinb. med. J., 57,

Doll, R., and Pygott, F. (1952). Factors influencing the rate of healing of gastric ulcers. Lancet, 1,171 .

- Jones, F. Avery, Pygott, F., and Stubbe, J. L. (1957). The risk of gastric cancer after medical treatment for gastric ulcer. Gastroenterologia (Basel), 88, 1.

Finer, D. I., and Fry, J. (1955). Peptic ulcer in general practice. Brit.

Flint, F. J., and Warrack, A. J. N. (1958). Acute peptic ulceration in emphysema. Lancet, 2, 178.

Jennings, D., and Richardson, J. E. (1954). Giant lesser-curve gastric ulcers. Ibid, 2, 343.

Jones, F. Avery (1957). Clinical and social problems of peptic ulcer. Brit. med.J., 1, 719.

- (1959). Personal communication.

Malmros, $\dot{H}$., and Hiertonn, T. (1949). A post-investigation of 687 medically treated cases of peptic ulcer. Acta med. scand., 133, 229.

Martin, L., and Lewis, N. (1949). Peptic ulcer cases reviewed after ten years. Lancet, $2,1115$.

Natvig, P., Römcke, O., and Svaar-Seljesaeter, O. (1943). Results of medical treatment of gastric and duodenal ulcer. Acta med. scand.,113, 444.

Pygott, F. (1958). In Jones, F. Avery. Modern Trends in GastroEnterology, p. 132. Butterworth, London.

Qvigstad, I., and Römcke, O. (1946). Post-investigation of medically treated gastric and duodenal ulcers. Acta med. scand., 126, 34

Steigmann, F. (1943). Considerations on the diagnosis of large gastric ulcers and implications as to treatment. Amer. J. dig. Dis, 10, 88.

Strange, S. L. (1959). Giant innocent gastric ulcer. Brit. med. J., 1 , 476.

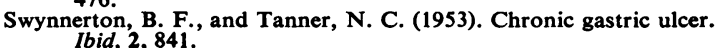

Visick, A. H. (1948). Measured radical gastrectomy. Lancet, 1, 505. 